\title{
ESTADO TÉRMICO DE PRODUTOS ARMAZENADOS EM SILOS COM SISTEMA DE AERAÇÃO: ESTUDO TEÓRICO E EXPERIMENTAL
}

\section{FABIANE A. DE OLIVEIRA ${ }^{1}$, OLEG A. KHATCHATOURIAN ${ }^{2}$, ANDERSON BIHAIN ${ }^{3}$}

\begin{abstract}
RESUMO: O resfriamento de grãos por ventilação de ar ambiente aplica-se amplamente nas etapas finais de secagem e para controle posterior da temperatura de grãos armazenados. O objetivo deste trabalho é realizar estudo teórico-experimental sobre o estado térmico de massa de grãos de soja sujeita à aeração. Foram obtidos dados experimentais sobre a dinâmica de resfriamento de massa pré-aquecida de grãos de soja para diferentes alturas da coluna de grãos e velocidades do ar. A análise dos resultados mostrou que a taxa de resfriamento varia significativamente durante todo o processo e em todo o domínio e a difusividade térmica das camadas não poderia ser considerada constante. Para simular a dinâmica de resfriamento, foram apresentados dois modelos matemáticos. No primeiro modelo, o domínio de resfriamento foi dividido pela fronteira móvel em duas zonas, representadas por diferentes difusividades térmicas (análogo de problema de Stefan). No outro modelo, todo o domínio foi dividido hipoteticamente em pequenas camadas e foi considerado que o processo de equilíbrio térmico entre o ar e a massa de grãos para essas camadas é atingido instantaneamente ("reatores homogêneos"). Os resultados de simulações mostraram concordância satisfatória com os dados experimentais.
\end{abstract}

PALAVRAS-CHAVE: armazenamento, aeração, resfriamento.

\section{THERMAL STATE OF STORED PRODUCTS IN STORAGE BINS WITH AERATION SYSTEM: EXPERIMENTAL-THEORETICAL STUDY}

\begin{abstract}
The cooling of grains by ventilation with ambient air applies widely in the final stages of drying and for the subsequent temperature control of stored grains. The objective of this work is to carry out an experimental-theoretical study on the thermal state of a soybean mass, subject to the aeration. The experimental data on cooling dynamics of preheated soybean mass were obtained for different heights of the grain column and air velocities. The analysis of the results showed that the cooling rate varies significantly during all process in all domains and the thermal diffusivity of the layers cannot be considered as a constant. To simulate the cooling dynamics two mathematical models were introduced. In the first model the cooling domain was divided by means of the movable frontier in two zones represented by different coefficients of thermal diffusivity (analogous of Stefan's problem). In another model all the domain was divided hypothetically in small layers and was considered that the thermal balance between the air and the mass of grains for these layers is reached instantly ("homogeneous reactors"). The results of simulations showed a satisfactory agreement with the experimental data.
\end{abstract}

KEYWORDS: storage, aeration, cooling.

\footnotetext{
${ }^{1}$ Física, Profa. Dra., Departamento de Física, Estatística e Matemática, UNIJUI, Ijuí - RS, Fone: (0XX55) 3332.0204, fabiane@unijui.tche.br.

${ }^{2}$ Eng $^{\circ}$ Mecânico, Prof. Doutor, Departamento de Física, Estatística e Matemática, UNIJUI, Ijuí - RS.

${ }^{3}$ Bolsista de Iniciação Científica - CNPq, UNIJUI.

Recebido pelo Conselho Editorial em: 7-6-2006

Aprovado pelo Conselho Editorial em: 22-3-2007
} 


\section{INTRODUÇÃO}

Durante o armazenamento de grãos em silos, esses ficam sujeitos às alterações da temperatura ambiente que, junto com o teor de água da massa de grãos, são responsáveis pela evolução da população de insetos e microorganismos que influenciam significativamente na qualidade do produto. Para preservar essa qualidade, utiliza-se o processo de aeração, que consiste no resfriamento dos grãos através da passagem forçada de ar ambiente pela massa de grãos.

Apesar do número considerável de recomendações sobre administração de armazéns, os projetistas e operadores de sistemas de armazenamento encontram várias dificuldades, desenvolvendo e operando os sistemas de aeração de grãos. A modelagem matemática adequada do funcionamento desses sistemas e a simulação da distribuição dos parâmetros em silos, como a pressão estática, a velocidade do ar e a temperatura poderiam facilitar significativamente o aperfeiçoamento do armazenamento.

Atualmente, existem modelos que descrevem satisfatoriamente o escoamento do ar em silos em condições isotérmicas (KHATCHATOURIAN \& SAVICKI, 2004). Ao mesmo tempo, a simulação do estado térmico de um silo para condições não-isotérmicas apresenta algumas dificuldades relacionadas com a ausência de dados confiáveis sobre coeficientes de transferência de calor entre o grão e o ar, coeficientes de difusividade térmica do próprio grão e da camada de grãos.

Vários autores tentaram descrever a dinâmica de variação de temperatura da massa de grãos. Alguns deles, como, por exemplo, BAKKER-ARKEMA \& BICKERT (1966) e KHATCHATOURIAN \& OLIVEIRA $(2005,2006)$, admitiram que o fenômeno de transferência de massa de água pode ser desprezado. Outros, como BAKKER-ARKEMA et al. (1974) e MONTROSS \& MAIER (2000), levaram em conta a transferência de calor e de massa em camadas de grãos. Esses últimos modelos seriam mais adequados para a simulação de secagem de grãos, apesar de, às vezes, estarem unidos pelo termo "Aeration models" (NAVARRO \& NOYES, 2002).

Os objetivos deste artigo são: obter dados experimentais da temperatura da massa de grãos de soja em função do tempo e da posição na coluna para diferentes velocidades do ar de aeração; desenvolver modelo matemático que descreva a dinâmica de resfriamento da massa de soja; realizar simulações numéricas utilizando dois modelos matemáticos e compará-las com os dados experimentais obtidos, e realizar a escolha do modelo que melhor se adapte e descreva o processo de resfriamento/aquecimento de grãos.

\section{MATERIAL E MÉTODOS}

\section{Estudo Experimental}

\section{Equipamento}

Os dados experimentais do resfriamento foram obtidos de um pequeno "silo" composto por um tubo de PVC (diâmetro de $0,15 \mathrm{~m}$ e 1,0 m de altura) com isolamento térmico nas laterais. $\mathrm{O}$ esquema da bancada é mostrado na Figura 1.

Os grãos de soja com teor de água médio de $12 \%$ (b.u) foram previamente selecionados e limpos e para a realização dos experimentos, e aquecidos em estufa até temperatura aproximada de $52{ }^{\circ} \mathrm{C}$ (temperatura inicial $-\mathrm{T}_{0}$ ), durante aproximadamente $8 \mathrm{~h}$, suficiente para uniformizar a temperatura em toda a massa de grãos.

O resfriamento dos grãos aquecidos foi realizado por meio da ventilação de uma camada de grãos de soja de $0,60 \mathrm{~m}$ de altura pelo ar ambiente, por um ventilador centrífugo, acionado por motor elétrico com $3 / 4 \mathrm{cv}$. Esse ventilador estava ligado a um inversor de freqüência que controlava as velocidades do ar do ventilador. O ar era impulsionado e passava por uma tubulação de $0,05 \mathrm{~m}$ de diâmetro onde uma placa de orifício, ligada a um manômetro, media a diferença de pressão do 
ar na placa. A partir dessa medida, foi possível calcular a vazão do ar que atravessava a placa, dada pela expressão abaixo:

$$
\mathrm{Q}=\mathrm{CA}_{0} \sqrt{\frac{2 \Delta \mathrm{p}}{\rho_{\mathrm{a}}}}
$$

em que,

$\mathrm{C}=0,72$ - coeficiente da placa de orifício calibrada utilizada nos experimentos;

$\rho_{\mathrm{a}}$ - densidade do ar;

$\Delta \mathrm{p}$ - diferença de pressão na placa medida pelo manômetro, e

$\mathrm{A}_{0}=0,001017 \mathrm{~m}^{2}$ - área do orifício da placa.

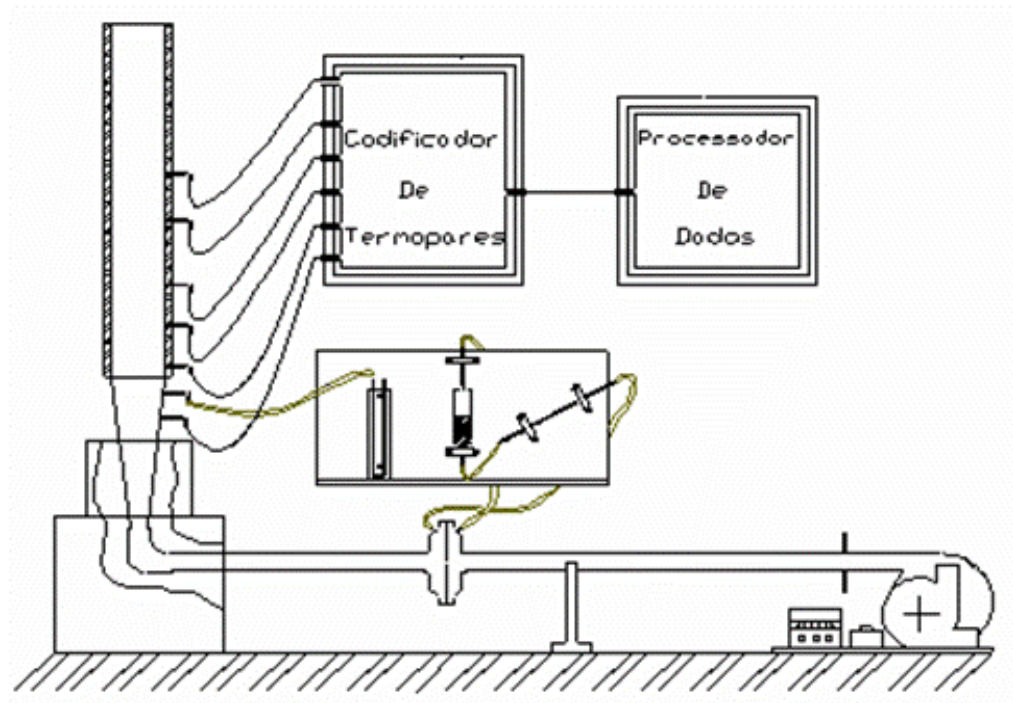

FIGURA 1. Esquema da bancada construída para estudar o resfriamento de grãos de soja.

Experimentos preliminares realizados na bancada para o intervalo de velocidades do ar estudadas neste trabalho $\left(0,05\right.$ a $\left.0,5 \mathrm{~m} \mathrm{~s}^{-1}\right)$ mostraram que a diferença da temperatura do ar e do grão durante o resfriamento não é significativa (excluindo a secção próxima da entrada do ar, nos instantes iniciais). Por isso, a temperatura dos grãos foi medida por termopares modelo MTK - tipo $\mathrm{K}$, inseridos dentro da massa de grãos ao longo do tubo, nas seguintes secções da coluna de grãos: $\mathrm{y}=0,15 \mathrm{~m} ; \mathrm{y}=0,27 \mathrm{~m} ; \mathrm{y}=0,40 \mathrm{~m} ; \mathrm{y}=0,54 \mathrm{~m}$ e ligados a uma placa de aquisição de dados A/D do tipo LR 7018, que permitia realizar até dez leituras por segundo. Na entrada da câmara, foi medida a temperatura do ar de entrada utilizado para aeração (temperatura ambiente - $\mathrm{T}_{\mathrm{a}}$ ).

Em condições reais, a diferença entre as temperaturas no interior dos silos e a temperatura do ar ambiente (utilizado para a aeração) raramente excedem $10{ }^{\circ} \mathrm{C}$, porém, nas experiências realizadas com resfriamento de grãos de soja, essa diferença foi mantida entre $20{ }^{\circ} \mathrm{C}$ e $25^{\circ} \mathrm{C}$, com o objetivo de aumentar a sensibilidade das medidas e reduzir o erro relativo.

\section{Resultados}

Na Figura 2, os pontos apresentam os dados experimentais da temperatura da massa de grãos de soja obtidos em diferentes secções do tubo para uma velocidade do ar na entrada do tubo constante.

A análise dos resultados mostra que a taxa de resfriamento varia significativamente durante todo o processo, em todas as secções. Para secções mais próximas da entrada do ar fresco, o resfriamento ocorre mais rápido. A dinâmica de resfriamento com diferentes velocidades para a distância y $=0,27$ m é mostrada na Figura 3 e possui o mesmo comportamento. 


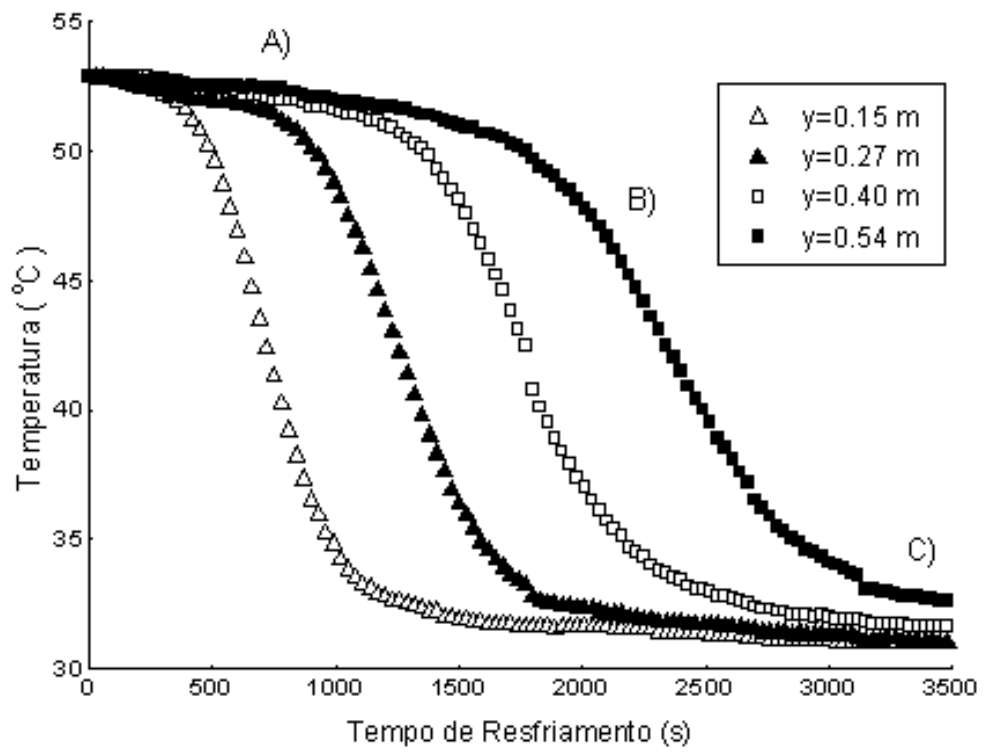

FIGURA 2. Dinâmica de resfriamento de massa de grãos de soja, para diferentes secções da coluna de grãos, para a velocidade do ar na entrada do tubo de $\mathrm{v}=0,23 \mathrm{~m} \mathrm{~s}^{-1}$ (dados experimentais).

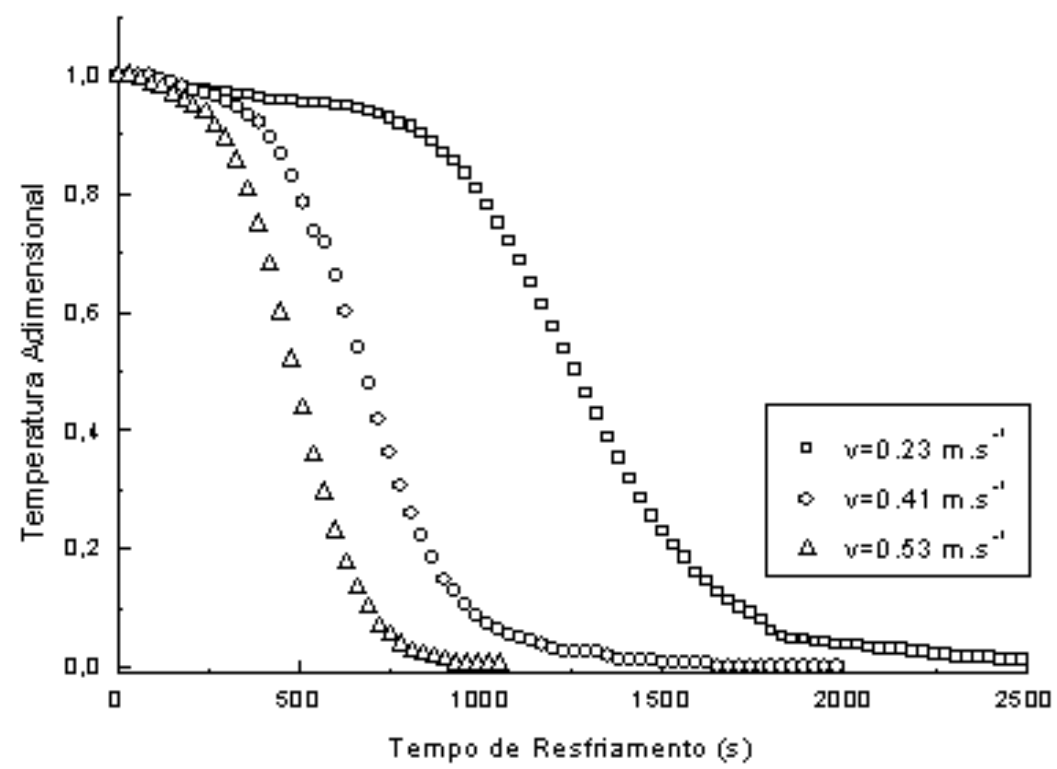

FIGURA 3. Temperatura adimensional $\left[\left(T-T_{a}\right) /\left(T_{0}-T_{a}\right)\right]$ para três diferentes velocidades do ar.

Para secções mais distantes da entrada, podem-se identificar três intervalos principais de variação de taxa de resfriamento: A) no primeiro intervalo (início do processo de resfriamento), a taxa de resfriamento não é muito alta (resfriamento lento). Isso ocorre porque a temperatura do ar que retira o calor dos grãos das secções anteriores, mais próximas à entrada de ar, cresce e reduz a capacidade do ar de resfriar as camadas mais distantes. A extensão desse intervalo depende da secção analisada e da velocidade do ar; B) no segundo intervalo, quando o resfriamento da massa de grãos nas secções iniciais já ocorreu, a taxa de resfriamento aumenta consideravelmente e, por consequiência, ocorre aceleração do processo de resfriamento das camadas mais distantes. A parte principal do processo de resfriamento encontra-se neste intervalo, e C) no terceiro intervalo, novamente ocorre resfriamento lento, onde a temperatura de grãos assintoticamente tende para a temperatura inicial do ar. 
No início, a camada considerada está sendo ventilada por ar aquecido pelas camadas anteriores, "esperando" a chegada do ar frio. À medida que ocorre o resfriamento das camadas anteriores, o ar que passa pela camada considerada possui a temperatura cada vez menor. A taxa de resfriamento dos grãos, que é proporcional à diferença entre as temperaturas do grão e do ar, aumenta. Posteriormente, essa diferença reduz-se devido à diminuição da temperatura do grão pelo resfriamento. Todos esses fatores implicam que a difusividade térmica do meio particulado (massa de grãos) varia com a temperatura, que, por sua vez, varia tanto com o tempo em cada secção quanto com a altura para cada instante de tempo considerado.

$\mathrm{O}$ aumento da velocidade do ar acelera o processo de resfriamento, fornecendo à massa de grãos mais ar frio por unidade do tempo (Figura 3). Os resultados obtidos permitiram avaliar dois modelos matemáticos do processo de resfriamento apresentados na próxima seção.

\section{Estudo Teórico}

\section{Modelos matemáticos}

Inicialmente, aplicando o modelo de estado térmico de uma barra com isolamento térmico lateral com temperatura fixa em uma das extremidades e com transferência de calor por convecção na outra, é possível mostrar que os resultados obtidos desse modelo não descrevem com boa concordância os dados experimentais, trabalhando com a difusividade térmica $\alpha$ e o coeficiente de transferência de calor $\mathrm{h}$ constantes durante todo o processo de resfriamento. Por outro lado, a variação desses coeficientes torna o problema não-linear e exige o conhecimento das dependências desses coeficientes com a temperatura.

Neste trabalho, estão apresentados dois modelos matemáticos que permitiram obter razoável concordância entre dados experimentais e simulados para a descrição da dinâmica de resfriamento de massa de grãos previamente aquecidos sujeitos à aeração à temperatura ambiente.

No primeiro modelo (modelo 1), para transformar o problema não-linear de propagação do calor numa seqüência de problemas lineares com contorno móvel, foi desenvolvido modelo matemático parecido com o problema de Stefan.

Seja o domínio $\Omega=\left\{(\mathrm{y}, \mathrm{t}) \mid 0<\mathrm{y}<\infty ; 0<\mathrm{t}<\mathrm{t}_{\max }\right\}$ dividido por dois subdomínios $\Omega^{2}=\left\{(\mathrm{y}, \mathrm{t}) \mid 0<\mathrm{y}<\mathrm{S}(\mathrm{t}) ; 0<\mathrm{t}<\mathrm{t}_{\max }\right\}$ e $\Omega^{1}=\left\{(y, t) \mid S(t)<y<\infty ; 0<t<t_{\max }\right\}$, em que $\mathrm{S}(\mathrm{t})$ é alguma fronteira móvel inicialmente desconhecida. Os subdomínios $\Omega^{1}$ e $\Omega^{2}$ possuem diferentes difusividades térmicas e as temperaturas $\mathrm{T}_{1}(\mathrm{y}, \mathrm{t})$ e $\mathrm{T}_{2}(\mathrm{y}, \mathrm{t})$ nesses subdomínios satisfazem às correspondentes equações de condução de calor:

$$
\begin{gathered}
\frac{\partial^{2} \mathrm{~T}_{1}}{\partial \mathrm{y}^{2}}=\frac{1}{\alpha_{1}} \frac{\partial \mathrm{T}_{1}}{\partial \mathrm{t}}, \quad(\mathrm{y}, \mathrm{t}) \in \Omega^{1} \\
\frac{\partial^{2} \mathrm{~T}_{2}}{\partial \mathrm{y}^{2}}=\frac{1}{\alpha_{2}} \frac{\partial \mathrm{T}_{2}}{\partial \mathrm{t}}, \quad(\mathrm{y}, \mathrm{t}) \in \Omega^{2}
\end{gathered}
$$

em que,

$\mathrm{T}(\mathrm{y}, \mathrm{t})$ - temperatura da massa de grãos;

y - posição na coluna de grãos, e

$\alpha$ - difusividade térmica da massa de grãos.

Um esquema do problema pode ser visualizado na Figura 4.

Conforme o modelo proposto e satisfazendo às condições de continuidade da solução, foi obtida a temperatura constante da fronteira móvel $\left(\mathrm{T}_{\mathrm{S}}\right)$ de separação dos dois domínios, como no Problema de Stefan. 


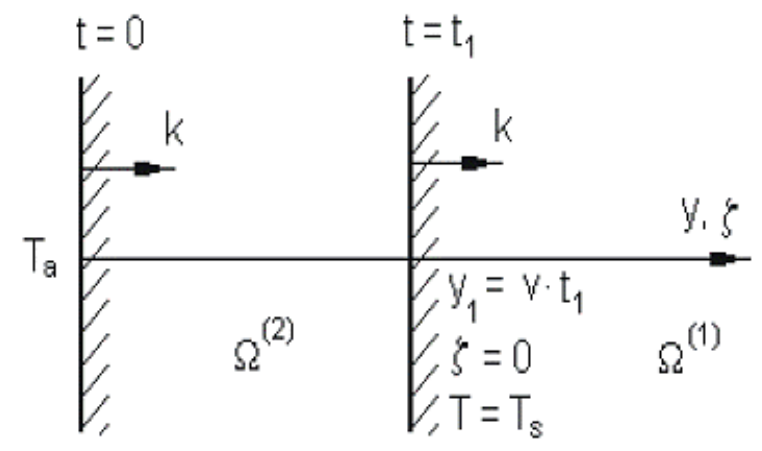

FIGURA 4. Esquema do problema-modelo 1.

A posição da fronteira móvel S, em função do tempo, foi escolhida na forma linear, dada pela expressão:

$$
\mathrm{S}(\mathrm{t})=\mathrm{kt}
$$

em que,

$\mathrm{k}$ - constante de proporcionalidade e representa a velocidade da frente de resfriamento que depende da velocidade do ar no tubo.

Conforme a posição dessa fronteira, a camada de grãos foi dividida em dois intervalos separados pela posição da fronteira.

À direita da fronteira, relacionando a posição da fronteira com a nova origem, foi introduzida uma nova coordenada dada por:

$$
\varsigma=\mathrm{y}-\mathrm{S}(\mathrm{t})
$$

Fazendo a mudança de variáveis, substituindo as expressões (4) e (5) na equação de condução de calor unidimensional (eq.2), tem-se :

$$
\frac{\mathrm{d}^{2} \mathrm{~T}_{1}(\varsigma)}{\mathrm{d} \varsigma^{2}}+\frac{\mathrm{k}}{\alpha_{1}} \frac{\mathrm{dT}_{1}(\varsigma)}{\mathrm{d} \zeta}=0
$$

As condições de contorno para esse caso são:

$$
\mathrm{T}_{1}(\varsigma=0)=\mathrm{T}_{\mathrm{S}} \quad \text { e } \quad \mathrm{T}_{1}(\varsigma \rightarrow \infty)=\mathrm{T}_{0}
$$

A temperatura inicial da massa de grãos $\left(T_{0}\right)$ é constante para toda a massa de grãos.

A solução da eq.(6) foi obtida na seguinte forma:

$$
\mathrm{T}_{1}(\mathrm{y}, \mathrm{t})=\mathrm{T}_{0}+\left(\mathrm{T}_{\mathrm{S}}-\mathrm{T}_{0}\right) \mathrm{e}^{-\frac{\mathrm{k}}{\alpha_{1}}[\mathrm{y}-\mathrm{S}(\mathrm{t})]}
$$

A distribuição da temperatura no intervalo à esquerda da fronteira pode ser descrita pela equação de condução de calor para uma barra limitada com extremos mantidos a temperaturas constantes:

$$
\mathrm{T}_{2}(0, \mathrm{t})=\mathrm{T}_{\mathrm{a}} \text { e } \mathrm{T}_{2}(\mathrm{~S}, \mathrm{t})=\mathrm{T}_{\mathrm{S}}
$$

A solução da eq.(3) foi obtida na forma:

$$
T_{2}(y, t)=T_{a}+\left(T_{S}-T_{a}\right) \frac{y}{S}+\sum_{n=0}^{\infty} B_{n} e^{-n^{2} \pi^{2} \alpha_{2} t / S^{2}} \operatorname{sen}(n \pi y / S)
$$

em que, 


$$
B_{n}=\frac{2}{n \pi}\left[T_{S}(-1)^{n}-T_{a}\right]+\frac{2}{S} \int_{0}^{S} \varphi(y) \operatorname{sen}(n \pi y / S) d y
$$

Para esse caso, $\varphi$ (y) é a distribuição inicial da temperatura.

$\mathrm{Na}$ fronteira que separa os subdomínios, estão satisfeitas as condições:

$$
T_{1}[S(t)-0, t]=T_{2}[(S(t)+0, t)]=T_{S}, \quad t \in\left[0, t_{\max }\right]
$$

O problema considerado é intermediário entre o problema de estado térmico de um corpo constituído por dois materiais diferentes (com fronteira fixa) e o problema de Stefan, no qual a fronteira móvel separa os domínios de fases diferentes. As difusividades térmicas de cada domínio foram escolhidas minimizando a discrepância entre os dados experimentais e simulados.

No segundo modelo (modelo 2), o estado térmico da massa de grãos sujeita à aeração foi calculado considerando a conservação de energia do sistema composto pelos grãos de soja e o ar que entra em contato com esses grãos. Para realizar os cálculos, todo o domínio de integração $\Omega=\left\{(y, t) \mid 0<y<\infty ; 0<t<t_{\max }\right\}$ foi dividido em elementos retangulares com nodos em pontos $\left(\mathrm{y}_{\mathrm{i}}, \mathrm{t}^{(\mathrm{n})}\right) \in \Omega ; \mathrm{i}=1,2, \ldots, \mathrm{M} ; \mathrm{n}=0,1, \ldots, \mathrm{N}$. Nesse caso, $\mathrm{M}$ representa o número de divisões espaciais e $\mathrm{N}$ o número de divisões temporais.

A função contínua desconhecida $\mathrm{T}=\mathrm{T}(\mathrm{y}, \mathrm{t})$ foi substituída por um conjunto de pontos isolados $\mathrm{T}_{\mathrm{i}}^{(\mathrm{n})} \cong \mathrm{T}\left(\mathrm{y}_{\mathrm{i}}, \mathrm{t}^{(\mathrm{n})}\right)$.
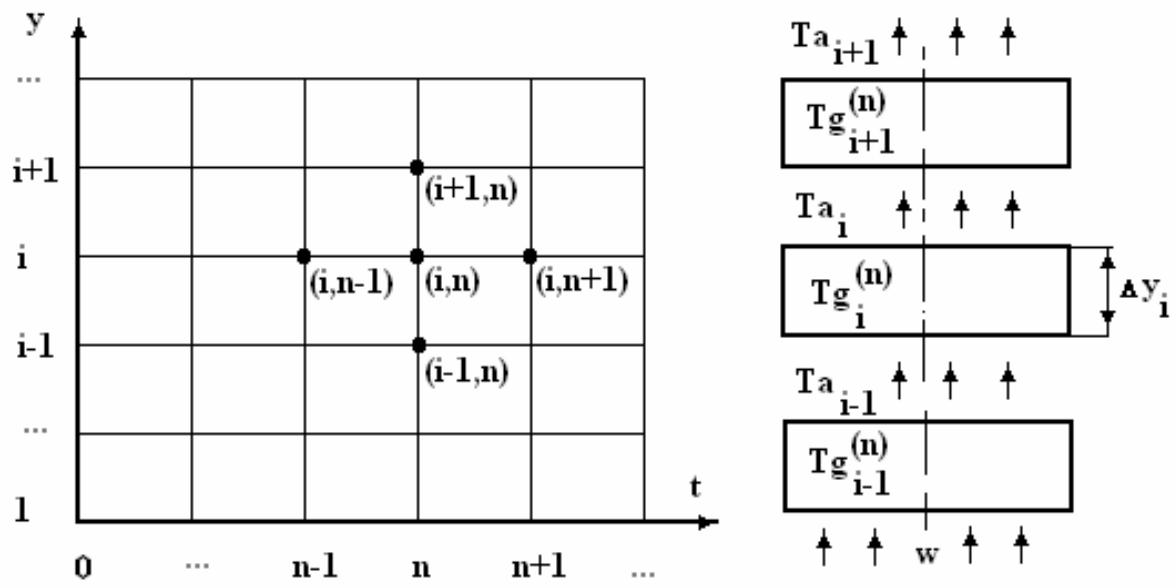

FIGURA 5. Discretização do domínio e esquema simplificado do resfriamento no modelo 2.

Admitindo que o equilíbrio térmico entre o ar e o grão se estabelece instantaneamente, isto é, o ar e os grãos em cada camada considerada sempre têm as temperaturas idênticas, a energia cedida pela massa de grãos em uma camada $\Delta y_{\mathrm{i}}$ por um tempo $\Delta t$ seria igual à energia recebida pelo ar. Dessa forma, pode-se escrever:

$$
c_{g} \rho_{g} \Delta y_{i} A(1-\varepsilon)\left(\operatorname{Tg}_{i}^{(n)}-\operatorname{Tg}_{i}^{(n+1)}\right)=c_{p a} \rho_{a} v A \varepsilon A \oint\left(\operatorname{Tg}_{i}^{(n+1)}-\frac{1}{\Delta t} \int_{t}^{t+\Delta t} \operatorname{Ta}_{i-1}(\tau) d \tau\right)
$$

Aplicando a regra de trapézios para o cálculo da integral:

$$
\frac{1}{\Delta t} \int_{t}^{t+\Delta t} \mathrm{Ta}_{\mathrm{i}-1}(\tau) \mathrm{d} \tau=\frac{\mathrm{Ta}_{\mathrm{i}-1}^{(\mathrm{n})}+\mathrm{Ta}_{\mathrm{i}-1}^{(\mathrm{n}+1)}}{2}
$$

que representa a temperatura média temporal do ar na entrada de camada " $i$ " no intervalo $\mathrm{t} \in\left[\mathrm{t}^{(\mathrm{n})}, \mathrm{t}^{(\mathrm{n}+1)}\right]$ e considerando $\operatorname{Ta}_{\mathrm{i}-1}^{(\mathrm{n})}=\operatorname{Tg}_{\mathrm{i}-1}^{(\mathrm{n})} ; \mathrm{Ta}_{\mathrm{i}-1}^{(\mathrm{n}+1)}=\operatorname{Tg}_{\mathrm{i}-1}^{(\mathrm{n}+1)}$, foi obtida a fórmula de 
recorrência para o cálculo da temperatura das camadas de grãos em correspondentes instantes de tempo (KHATCHATOURIAN \& OLIVEIRA, 2006):

$$
\operatorname{Tg}_{\mathrm{i}}^{(\mathrm{n}+1)}=\frac{\mathrm{c}_{\mathrm{g}} \rho_{\mathrm{g}} \Delta \mathrm{y}_{\mathrm{i}}(1-\varepsilon) \operatorname{Tg}_{\mathrm{i}}^{(\mathrm{n})}+\mathrm{c}_{\mathrm{pa}} \rho_{\mathrm{a}} \mathrm{v} \varepsilon \Delta \mathrm{t}\left(\operatorname{Tg}_{\mathrm{i}-1}^{(\mathrm{n})}+\operatorname{Tg}_{\mathrm{i}-1}^{(\mathrm{n}+1)}\right) / 2}{\mathrm{c}_{\mathrm{g}} \rho_{\mathrm{g}} \Delta \mathrm{y}_{\mathrm{i}}(1-\varepsilon)+\mathrm{c}_{\mathrm{pa}} \rho_{\mathrm{a}} \mathrm{v} \varepsilon \Delta \mathrm{t}},
$$

sendo $\mathrm{i}=1,2, \ldots, \mathrm{Me} \mathrm{n}=0,1,2, \ldots, \mathrm{N}$. Os índices $a$ e $g$ nas eqs.(13), (14) e (15) referem-se, respectivamente, ao ar e à camada de grãos de soja. Para as propriedades da camada de grãos $\left(c_{\mathrm{g}}, \rho_{\mathrm{g}}\right.$ e $\left.\varepsilon\right)$, foram utilizados os dados de MOHSENIN (1980), citado por ANDRADE et al. (2001).

Análogo ao cálculo de parâmetros de reatores homogêneos, as camadas obtidas pela divisão espacial foram chamadas de "reatores homogêneos" (KHATCHATOURIAN \& OLIVEIRA, 2006).

\section{RESULTADOS E DISCUSSÃO}

Aplicando os modelos apresentados, foram realizadas as simulações numéricas do processo de resfriamento da massa de grãos para diferentes velocidades do ar.

Para realizar as simulações utilizando o modelo 1 , foi necessário determinar os valores das difusividades térmicas de cada domínio, a posição da fronteira móvel e os valores da temperatura nessa fronteira para cada velocidade do ar estudada. Esses valores foram obtidos a partir da análise dos dados experimentais.

As simulações da posição da fronteira móvel $S(t)$ foram realizadas utilizando a eq.(4), na qual os valores de k foram calculados pelo Método de Mínimos Quadrados (M.M.Q), cuja variação com a velocidade do ar é mostrada na Figura 7. Pelos resultados da simulação apresentados na Figura 6, pode-se notar que, conforme a hipótese inicial [eq.(4)], a posição da fronteira varia linearmente com o tempo e depende da velocidade do ar.

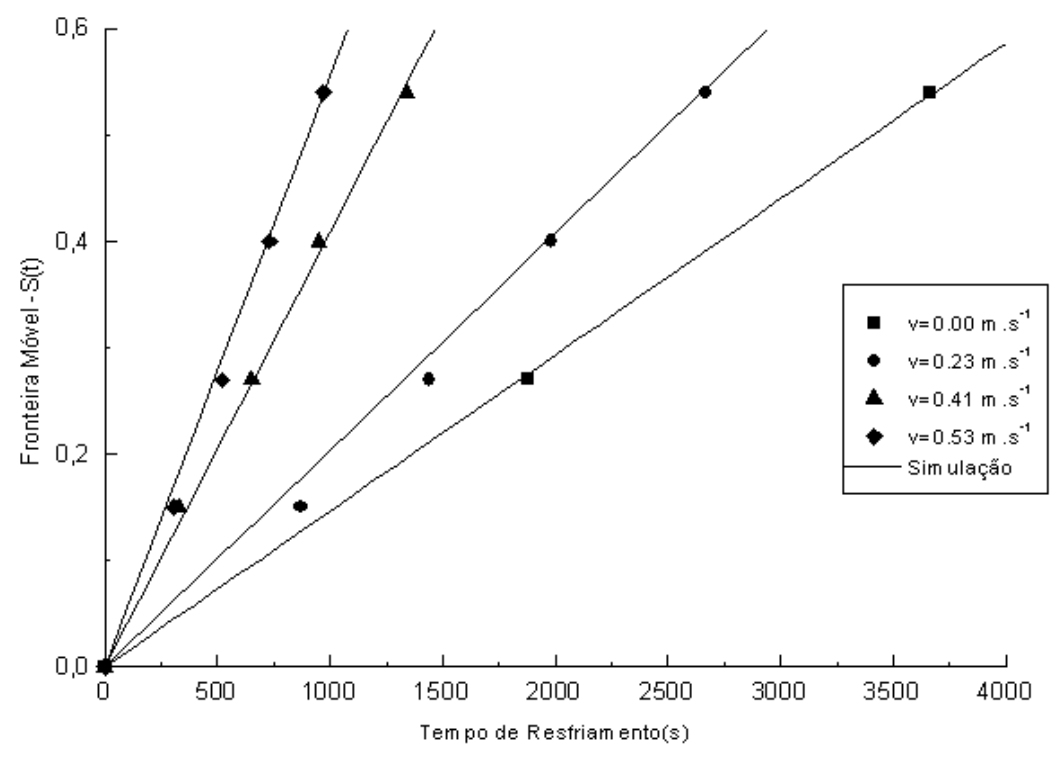

FIGURA 6. Posição da fronteira $S(t)$ em função do tempo para diferentes velocidades do ar.

A relação entre a velocidade da frente de resfriamento (velocidade da fronteira) e a velocidade do ar é mostrada na Figura 7. 


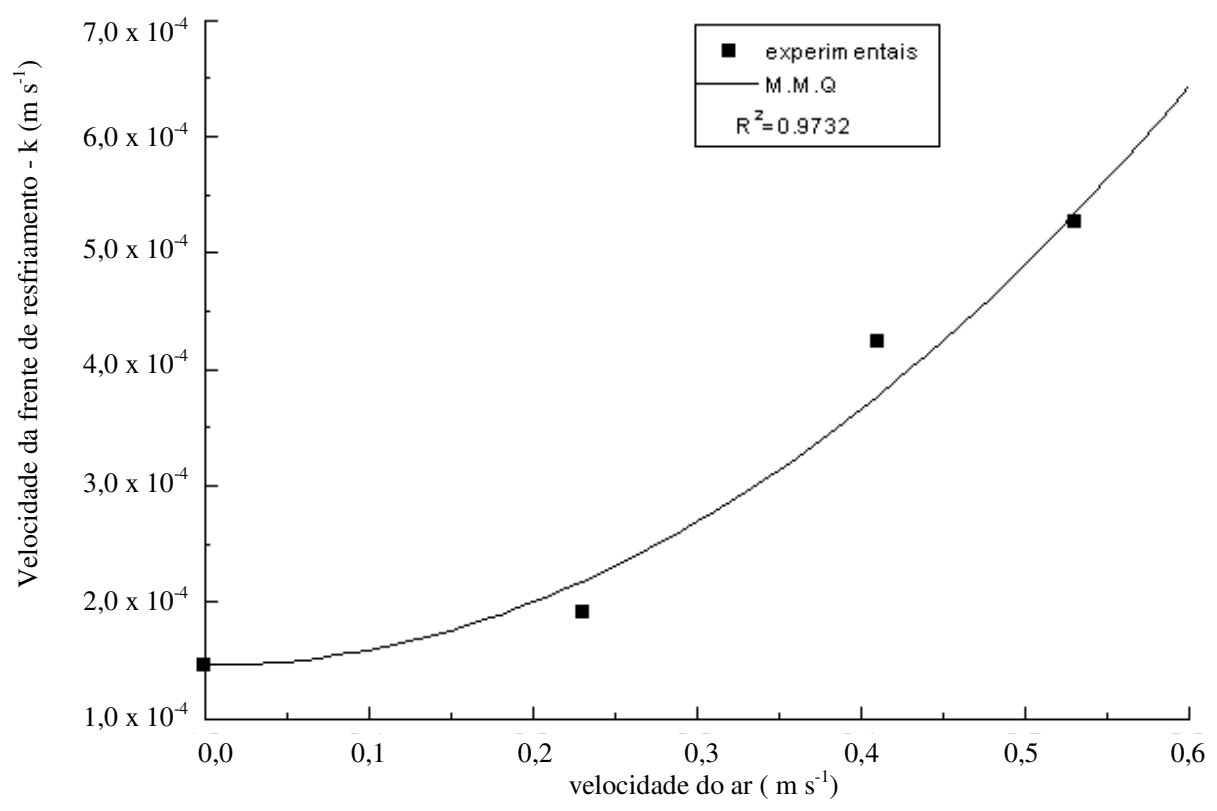

FIGURA 7. Velocidade da frente de resfriamento em função da velocidade do ar.

Na Figura 8, são mostrados os valores da difusividade térmica do primeiro e do segundo domínios para diferentes velocidades do ar.

Pode-se observar que a difusividade térmica $\alpha_{1}$ do primeiro domínio é maior que $\alpha_{2}$ do segundo domínio. Com aumento de velocidade, os valores absolutos de coeficientes $\alpha_{1}$ e $\alpha_{2}$ crescem.

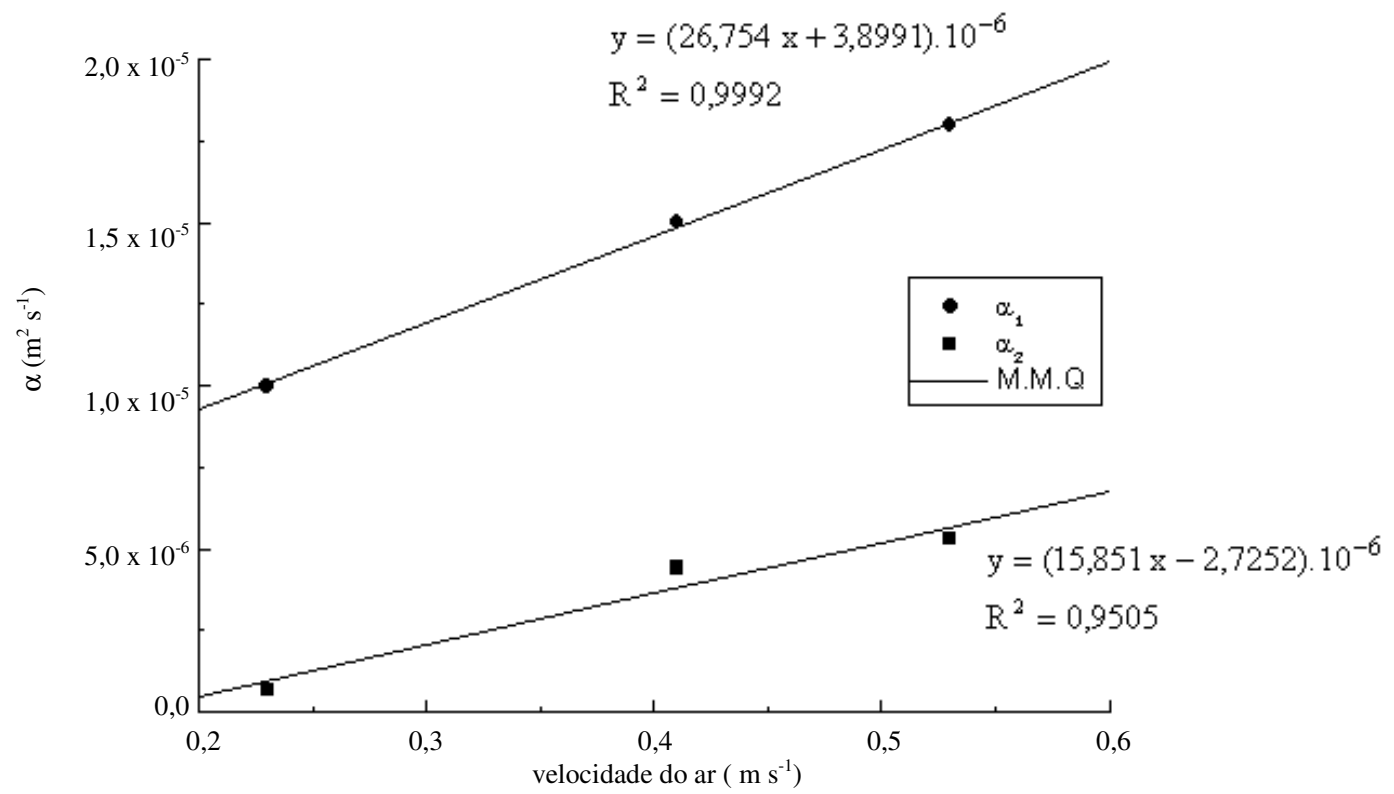

FIGURA 8. Valores de $\alpha_{1}$ e $\alpha_{2}$ para diferentes velocidades do ar.

A comparação com dados experimentais (Figuras 2 e 3 ) mostrou que tanto o primeiro como o segundo modelo descrevem razoavelmente o processo de resfriamento.

Uma discrepância entre os dados experimentais e calculados, no caso do primeiro modelo, é causada, provavelmente, pela linearização do modelo, escolhendo as difusividades térmicas constantes. Na Figura 10, são mostradas as curvas obtidas pelos dois modelos teóricos junto com os dados experimentais para os valores da temperatura adimensional ao longo da coluna de grãos para um tempo fixo. 


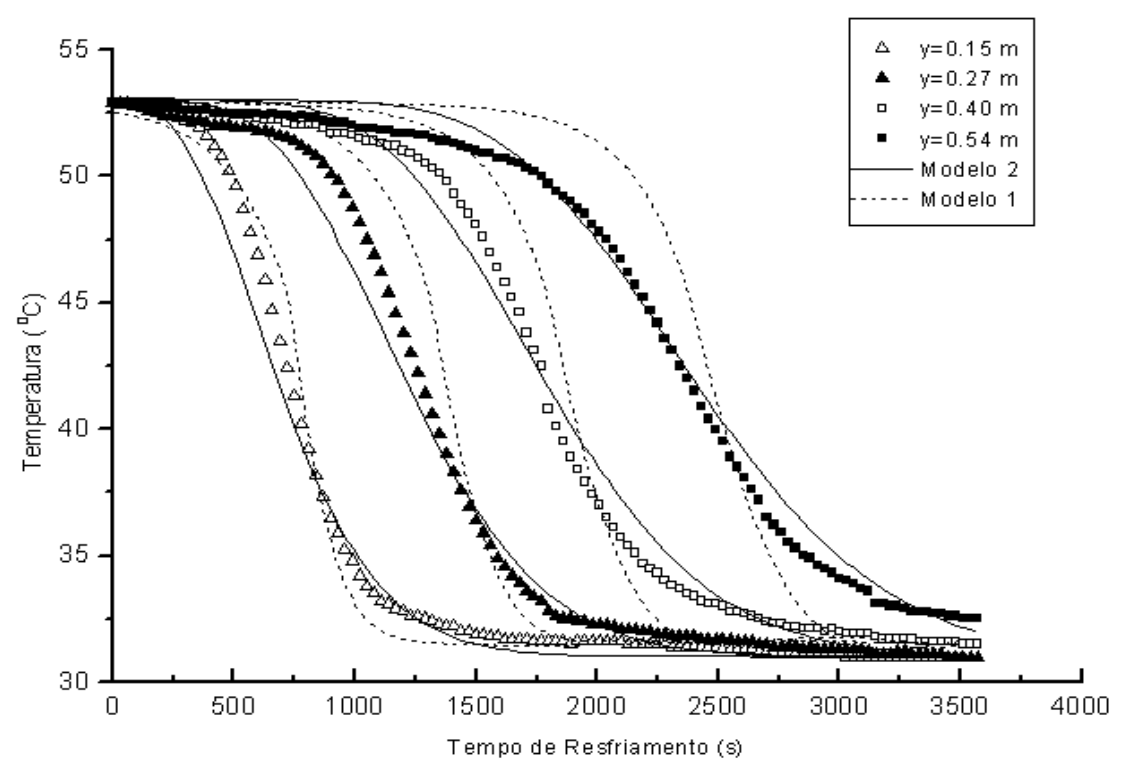

FIGURA 9. Dinâmica de resfriamento de massa de grãos de soja, para diferentes secções na coluna de grãos, para velocidade do ar de $0,23 \mathrm{~m} \mathrm{~s}^{-1}$.

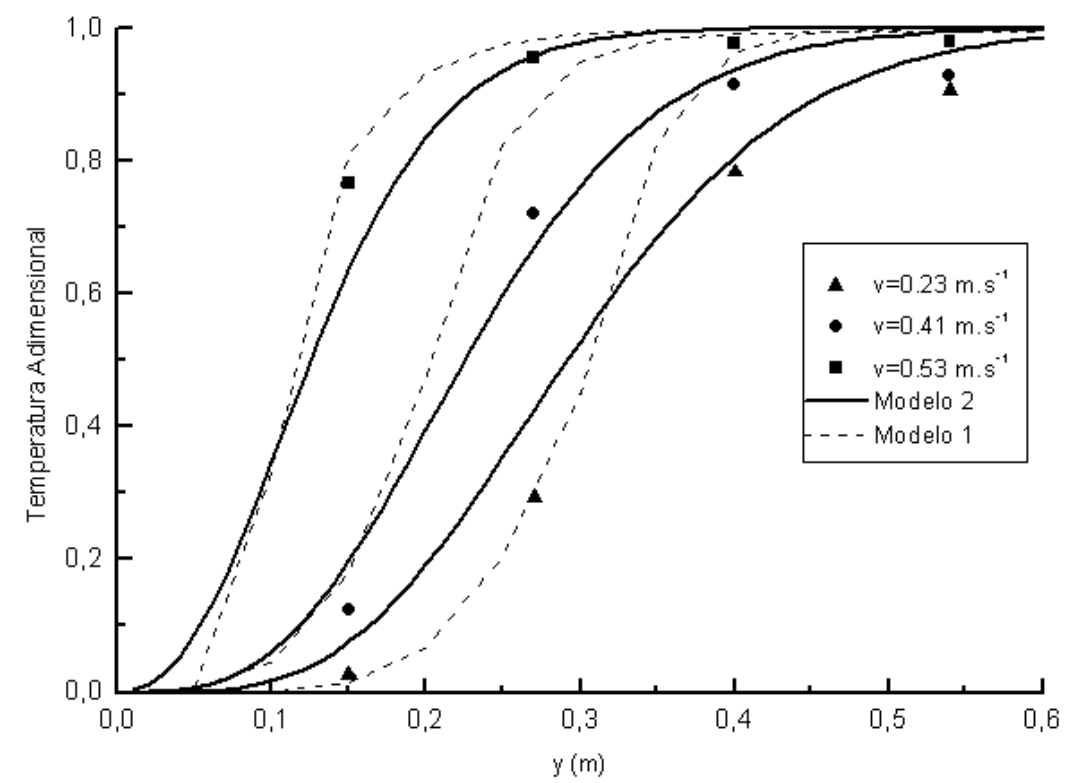

FIGURA 10. Temperatura adimensional ao longo da coluna de grãos, para diferentes velocidades do ar para $\mathrm{t}=570 \mathrm{~s}$.

No segundo modelo, os passos temporal $\Delta t=2 \mathrm{~s}$ e espacial $\Delta y_{\mathrm{i}}=0,01 \mathrm{~m}$ foram escolhidos de maneira que a diminuição posterior deles não influa sobre o resultado dos cálculos. As simulações mostraram que esse modelo tem melhor desempenho em comparação com o outro para o intervalo estudado de variação de velocidade. Foi calculado o coeficiente de correlação $\left(\mathrm{R}^{2}\right)$ para cada curva da Figura 9. Os valores obtidos para o modelo 1 estão no intervalo entre 0,9676 e 0,9897, e para o modelo 2 , entre 0,9919 e 0,9957 .

Uma boa coincidência entre a simulação utilizando o modelo 2 e os dados experimentais aprova a hipótese de equilíbrio térmico. Isso significa que o valor da difusividade térmica do próprio grão é bastante alto para que o tempo de permanência do ar na mesma camada (que depende da velocidade) seja compatível com o tempo característico de aquecimento do grão (que depende da difusividade térmica). Era esperado que o aumento da velocidade atrasasse o resfriamento da parte periférica do grão, o que levaria parte do ar a passar sem aquecimento 
completo, de acordo com o modelo de reator homogêneo. Nesse caso, a temperatura simulada de resfriamento deveria ser menor que a temperatura experimental da massa de grãos. $\mathrm{O}$ efeito contrário foi observado na Figura 9, indicando que, para a posição escolhida da junção do termopar no silo, na realidade, foi medida a temperatura da superfície do grão, sendo essa igual à temperatura do ar. Essa temperatura é sempre menor que a temperatura média do grão, por isso as temperaturas simuladas ficaram acima das obtidas nos experimentos. Para evitar (ou reduzir) essa discordância, o modelo pode ser complementado por um coeficiente de não-uniformidade (que varia entre 0 e 1). Uma grande vantagem desse modelo simples é a possibilidade de realizar as simulações em silos com secção transversal variável. Nesse caso, o campo de velocidades tem de ser calculado previamente (KHATCHATOURIAN \& SAVICKI, 2004).

\section{CONCLUSÕES}

O coeficiente de difusividade térmica das camadas não pode ser considerado constante, variando com o tempo, durante o resfriamento.

Foi mostrado que a divisão de toda a câmara pela fronteira móvel em dois domínios com coeficientes de difusividade térmica diferentes, permite resolver o problema do estado térmico nessas condições. Foi mostrado que a posição da fronteira móvel depende da velocidade do ar e varia linearmente com o tempo. Foram encontradas as dependências da difusividade térmica do primeiro e segundo domínios para diferentes velocidades do ar.

Foi proposto um modelo ("reatores homogêneos") que permitiu evitar a utilização da difusividade térmica para a simulação do resfriamento no intervalo estudado de variação dos parâmetros.

A comparação das simulações com dados experimentais mostrou que ambos os modelos descrevem razoavelmente o processo de resfriamento.

A aplicação do segundo modelo para simulação do estado térmico de um silo é preferível pela melhor coincidência com os dados experimentais e pela facilidade de considerar as secções transversais variáveis.

\section{REFERENCIAS}

ANDRADE, E.T.; MOLIN, L.; COUTO, S.M.; CARDOSO, G.E. Distribuição de temperatura em grãos armazenados em silos: modelagem por elementos finitos. Revista Brasileira de Armazenamento, Viçosa, n.26, p.28-35, 2001.

BAKKER-ARKEMA, F.W.; BICKERT, W.G. A deep-bed computational cooling procedure for biological products. Transactions of the ASAE, St. Joseph, v.9, n.6, p.834-6, 1966.

BAKKER-ARKEMA, F.W.; LEREW, L.E.; DEBOER, S.F.; ROTH, M.G. Grain dryer simulation. Agricultural Experiment Station Report. East Lansing: Michigan State University, 1974. n.224. $80 \mathrm{p}$.

KHATCHATOURIAN, O.A.; SAVICKI, D.L. Mathematical modelling of airflow in an aerated soya bean store under non-uniform conditions. Biosystems Engineering, Edinburg, v.88, n.2, p.20111, 2004.

KHATCHATOURIAN, O.A.; de OLIVEIRA, F.A. Mathematical modelling of air flow and thermal state in large aerated grain storage. In: INTER-AMERICAN DRYING CONFERENCE, 3., 2005, Montreal. Proceedings... Montreal: McGill University, 2005. 1 CD-ROM.

KHATCHATOURIAN, O.A.; de OLIVEIRA, F.A. Mathematical modelling of airflow and thermal state in large aerated grain storage. Biosystems Engineering, Edinburg, v.95, n.2, p.159-69, 2006.

MOHSENIN, N.N. Thermal properties of foods and agricultural materials. London: Gordon \& Breach Publishers, 1980. 404 p. 
MONTROSS, M.D.; MAIER, D.E. Simulated performance of conventional high-temperature drying, dryaeration and combination drying of shelled corn with automatic conditioning. Transactions of the ASAE, St. Joseph, v.43, n.3, p.691-9, 2000.

NAVARRO, S.; NOYES, R.T. (Eds.). The mechanics and physics of modern grain aeration management. New York: CRC Press, 2002. 647 p. 